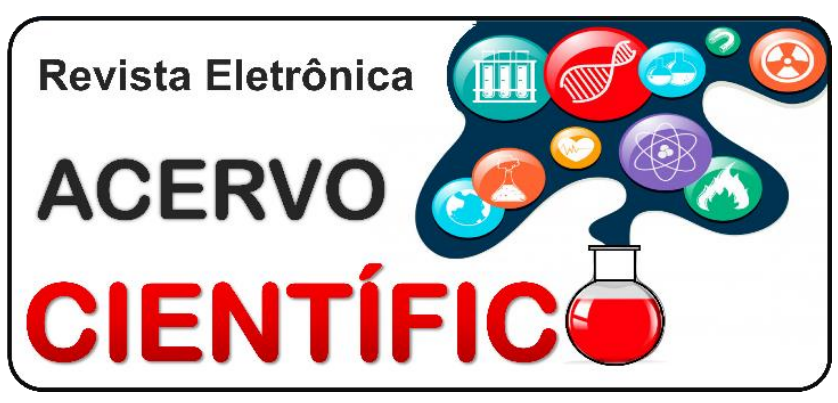

\title{
REVISÃO BIBLIOGRÁFICA
}

Recebido em: 10/2020

Aceito em: 11/2020

Publicado em: 12/2020

\section{Psicodermatoses: uma análise dos aspectos fisiopatológicos, sociais e dos tratamentos multidisciplinares}

Psychodermatoses: an analysis of physiopathological, social aspects and multidisciplinary treatments

Psicodermatosis: análisis de aspectos fisiopatológicos, sociales y tratamientos multidisciplinarios

Ingrid Hovsepian de Souza ${ }^{1 *}$, Milena Ferreira Gandra², Priscila Avelina Pereira Ribeiro², Bárbara Camargo da Rocha Pereira ${ }^{3}$, Cecília Gonçalves de Paula ${ }^{3}$, Clara Borges Pires ${ }^{3}$, Tainara Vieira de Amorim $^{4}$, Thayná Gomes de Aguiar ${ }^{5}$, Maria Eduarda Vilela Amarante ${ }^{6}$, Príncia Christino de Abreu Carvalho ${ }^{7}$.

Resumo: Este estudo buscou aprofundar, por meio de uma sistemática revisão bibliográfica, a respeito da psicodermatologia, que trata-se da união da Dermatologia e Psiquiatria, com o intuito de cessar um ciclo vicioso ao qual a existência de distúrbios psicológicos geram alterações cutâneas e manifestações dermatológicas são capazes de levar a alterações psíquicas. Neste ínterim, após uma situação de estresse, por meio de diferentes mecanismos, é desencadeada a liberação de diversos neuropeptídeos e neurotrofinas, que possuem efeitos pró-inflamatórios, principalmente através da ativação de mastócitos, levando a uma inflamação neurogênica. Por conseguinte, com o surgimento de dermatoses, a autoconfiança fica comprometida podendo acarretar o sofrimento psíquico, uma vez que a aparência física é a primeira impressão no contato humano sendo uma importante forma de comunicação. Visto sua importância e impacto psicossocial a abordagem terapêutica deve ser multidisciplinar, envolvendo médicos da atenção primária, dermatologista, psiquiatra e psicólogo, com o intuito de possibilitar o tratamento das psicodermatoses em sua total complexidade.

Palavras-chave: Dermatopatias, Estresse psicológico, Medicina integrativa.

Abstract: This study sought to deepen, through a systematic bibliographic review, about psychodermatology, which is the union of Dermatology and Psychiatry, in order to end a vicious cycle in which the existence of psychological disorders generate skin changes and dermatological manifestations are capable of leading to psychic changes. In the meantime, after a stressful situation, by means of different mechanisms, the release

\footnotetext{
${ }^{1}$ Universidade de Uberaba (UNIUBE), Uberaba - MG. `E-mail: ingridhovsepian1997@gmail.com

2 Centro Universitário de Caratinga, Caratinga - MG

${ }^{3}$ Centro Universitário de Belo Horizonte (UNIBH), Belo Horizonte - MG.

4 Universidad Central del Paraguay (UCP), Ciudad del Este - PY.

${ }^{5}$ Faculdade Dinâmica do Vale do Piranga, Ponte Nova - MG.

6 Universidade José do Rosário Vellano (UNIFENAS), Alfenas - MG.

${ }^{7}$ União educacional do Vale do Aço (UNIVAÇO), Ipatinga - MG.
} 
of several neuropeptides and neurotrophins is triggered, which have pro-inflammatory effects, mainly through the activation of mast cells, leading to neurogenic inflammation. Therefore, with the appearance of dermatoses, a self-confidence is compromised, which can cause psychological distress, since physical appearance is the first impression in human contact and is an important form of communication. Given its importance and psychosocial impact, a therapeutic approach must be multidisciplinary, involving primary care physicians, dermatologists, psychiatrists and psychologists, in order to enable the treatment of psychodermatoses in their total complexity.

Keywords: Skin diseases, Psychological stress, Integrative medicine.

Resumen: Este estudio buscó profundizar, a través de una revisión bibliográfica sistemática, sobre la psicodermatología, que es la unión de la Dermatología y la Psiquiatría, con el objetivo de poner fin a un círculo vicioso en el que la existencia de trastornos psicológicos generan cambios cutáneos y manifestaciones dermatológicas. son capaces de provocar cambios psíquicos. Mientras tanto, tras una situación estresante, mediante diferentes mecanismos, se desencadena la liberación de varios neuropéptidos y neurotrofinas, que tienen efectos proinflamatorios, principalmente a través de la activación de mastocitos, lo que conduce a la inflamación neurogénica. Por tanto, con la aparición de las dermatosis, la autoconfianza se ve comprometida y puede provocar malestar psicológico, ya que la apariencia física es la primera impresión en el contacto humano y es una forma importante de comunicación. Dada su importancia e impacto psicosocial, el abordaje terapéutico debe ser multidisciplinario, involucrando a médicos de atención primaria, dermatólogos, psiquiatras y psicólogos, a fin de posibilitar el tratamiento de las psicodermatosis en su total complejidad.

Palabras clave: Enfermedades de la piel, Estrés psicológico, Medicina integrativa.

\section{INTRODUÇÃO}

A pele é um órgão que tem uma função primária na receptividade tátil e, através da sua rica inervação, bem como a dos seus apêndices, ela se torna muito suscetível a reagir a estímulos emocionais (FRANÇA K, et al., 2013; GUPTA MA, 2018).

O estresse psicológico pode ser responsável por exacerbações de doenças dermatológicas imunomediadas, como a psoríase e a alopecia areata, bem como por gerar sintomas mediados por um estímulo adrenérgico, como o prurido (GUPTA MA, 2018). Nesse sentido, a psicodermatologia, uma ciência integrativa, foi criada para abordar a conexão entre a mente e a pele. Três campos principais estão envolvidos: psiquiatria, psicologia e dermatologia (FRANÇA K, et al., 2013).

O sistema nervoso e a pele têm uma ligação direta de origem, ambos derivam da ectoderme. A pele é inervada por terminações do sistema sensorial e do sistema nervoso autônomo. A relação entre nervos, células cutâneas, células imunitárias e secreções de neurotransmissores, neuropeptídeos, neurormônios e citocinas indicam que existe uma rede neuroimunocutâneoendócrina, em que se processa a ligação entre mente e pele (AZAMBUJA R, 2000).

O estresse psicológico é acompanhado por diversas respostas psicológicas e fisiológicas que afetam o sistema neuroimune e mobilizam o sistema neuroendócrino, influenciando na resposta inflamatória do corpo (ANTUNES J, 2019). Pacientes que relatam estresse psicológico constante em suas vida exibem mais sintomas dermatológicos e sintomas articulares do que aqueles que relatam sofrerem pouca influência do estresse. Porém mais estudos são necessários para avaliar melhor essa relação. (YANG HE ZHENG J). Por essa perspectiva, o estresse possui impacto sobre a pele, gerando uma série de alterações, doenças psicocutâneas e dermatoses.

Algumas consequências do estresse sobre a pele são: vasoconstrição ou vasodilatação, aumento da fragilidade capilar, prurido, psoríase, dermatite atópica, vitiligo e certas urticárias. Cada pessoa, ao expressar na pele seu estado interior, vive uma condição exclusiva, dependente da sua habilidade em lidar com fatores de tensão (AZAMBUJA R, 2000). Determinadas experiências de vida estressantes vêm sendo associadas ao 
início e/ou agravamento de sintomas em doenças de ordem inflamatória em que as citocinas desempenham um papel crucial, como a Psoríase (ANTUNES J, 2019).

A abordagem terapêutica dos distúrbios psicodermatológicos deve ser multidisciplinar, envolvendo médicos da atenção primária, dermatologista, psiquiatra e psicólogo. Os tratamentos incluem métodos cognitivo-comportamentais, drogas psicotrópicas, ervas e suplementos alternativos, o efeito placebo, hipnose e biofeedback. Quando os procedimentos individuais não produzem os resultados desejados, as combinações de medicamentos ou a adição de terapias não medicamentosas podem ter mais sucesso. $O$ tratamento de transtornos psiquiátricos normalmente resulta na cura dos distúrbios dermatológicos associados (SHENEFELT PD, 2011).

O objetivo do presente estudo consiste em analisar a importância da avaliação multidisciplinar dos indivíduos afetados pelas psicodermatoses, buscando a relação dos fatores psicológicos e biológicos em situações de estresse.

\section{REVISÃO BIBLIOGRÁFICA}

\section{Psicodermatologia}

A psicodermatologia trata-se da união da Dermatologia e Psiquiatria, com o intuito de cessar um ciclo vicioso ao qual a existência de distúrbios psicológicos geram alterações cutâneas, e manifestações dermatológicas são capazes de levar a alterações psíquicas (FRANÇA K, et al., 2013).Neste contexto percebemos a existência de conteúdos psíquicos profundos e confusos, que ao não se expressarem a nível verbal se manifestam a nível mental (MÜLLER MC E RAMOS DG, 2004).

Assim, é necessário que haja um entendimento profundo a respeito da classificação dos transtornos psicocutâneos para melhor escolha da terapêutica a ser seguida. Ao qual se dividem em: a) doenças psicofisiológicas pedidos; b) transtornos psiquiátricos com sintomas dermatológicos, e c) desordens dermatológicas com sintomas psiquiátricos. Além disso, devemos classificar também de acordo com sua intensidade e duração, sendo que, o estresse psicossocial agudo pode variar de minutos a horas, enquanto o estresse crônico se perpetua por vários dias, semanas ou meses (HUNTER HJA, et al., 2015).

Diante disso, a psicodermatologia busca entender tais alterações, sendo fundamental a realização de um trabalho conjunto a fim de integrar o tratamento, oferecendo resultados mais amplos, efetivos e duradouros com objetivo de melhorar a qualidade de vida desses pacientes (TOHID H, et al., 2019).

\section{Fisiopatologia}

A pele é a maior barreira do corpo contra o meio externo (ALEXOPOULOS A, 2016). E, através da sua rica inervação, a pele é conectada a estruturas centrais neuroendócrinas (PETERS EMJ, 2016). Quando um indivíduo é submetido ao estresse, o eixo hipotálamo-pituitária-adrenal (HPA) é ativado, ocorrendo a produção e liberação do hormônio liberador de corticotrofina $(\mathrm{CRH})$. O $\mathrm{CRH}$ irá atuar na adenohipófise aumentando a expressão gênica e secreção de pró-opiomelanocortina (POMC), um precursor polipeptídico que será convertido em outros peptídeos como a $\beta$-endorfina, o hormônio estimulante de $\alpha$-melanócitos ( $\alpha-\mathrm{MSH}$ ) e o hormônio adrenocorticotrófico (ACTH). O ACTH liga-se aos receptores de melanocortinas no córtex da adrenal, estimulando a síntese de glicocorticóides, que atuarão na circulação sistêmica. Os glicocorticóides, por sua vez, influenciam na transcrição de genes anti-inflamatórios (HUNTER HJA, 2015). Estudos recentes demonstraram que células da pele humana, como os queratinócitos da epiderme e os foliculares, as células de Langerhans, os melanócitos e os fibroblastos, possuem receptores para as substâncias mediadoras do eixo HPA (LEE CM, 2019).

Outra via que atua em resposta ao estresse é o eixo simpático-adrenal-medular (SAM). Ao ocorrer a exposição a situações de estresse, a medula adrenal libera epinefrina e norepinefrina, que irão atuar alterando funções imunológicas e inflamatórias, como a circulação, a proliferação e a produção de citocinas (ALEXOPOULOS A, 2016). Existe, ainda, um eixo cutâneo periférico que age de forma semelhante ao eixo HPA central, possuindo a capacidade de produzir peptídeos derivados da POMC (HUNTER HJA, 2015). As vias ativadas pelo estresse psicológico estão representadas na Figura 1. 
Figura 1 - Vias neuroendócrinas envolvidas na resposta cutânea ao estresse psicológico.

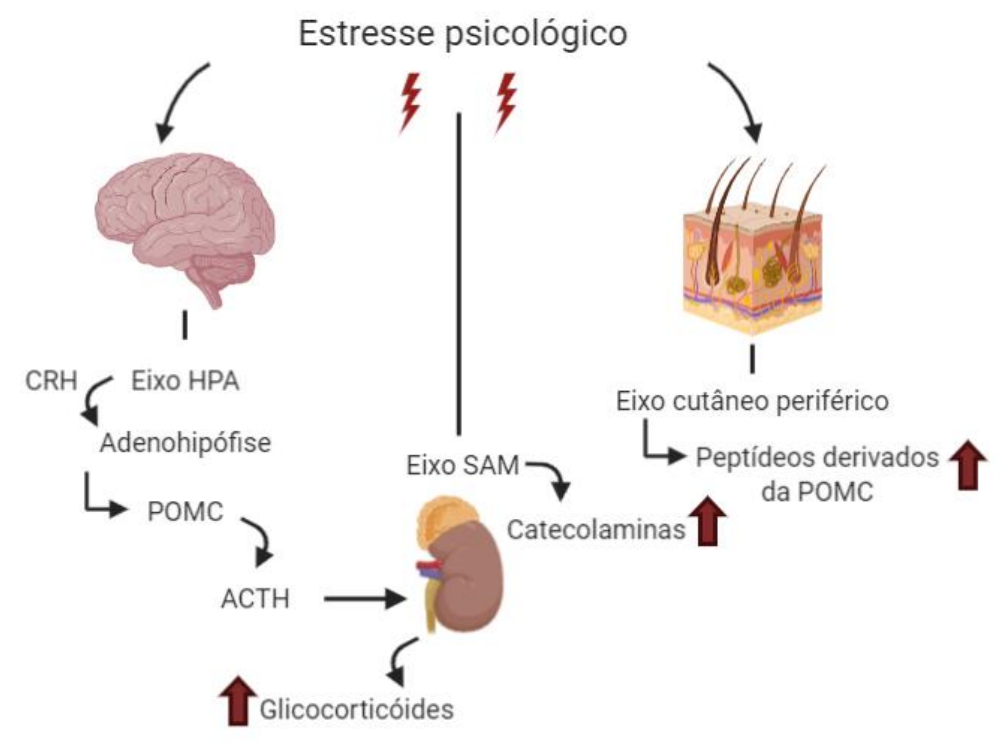

Fonte: Souza IH, et al., 2020. Baseada em The Impact of Psychosocial Stress on Healthy Skin, 2014.

Praticamente todas as células do sistema imune possuem receptores para as substâncias liberadas relacionadas ao estresse citadas acima. Tais substâncias atuam no aumento do tráfico, maturação e função dos granulócitos, linfócitos e de células dendríticas. O estresse agudo induz uma resposta imunológica do tipo Th1, com a produção de citocinas como a interleucina 2 (IL-2), o interferon-gama (INF-Y) e o fator de necrose tumoral alfa (TNF- $\alpha$ ). Já o estresse crônico induz uma troca para a resposta imune do tipo Th2, com a liberação de IL-4, IL-10 e IL-13 (HUNTER HJA, 2015; MOLLANAZAR NK, 2016). Este último mecanismo é o responsável pela patogênese do prurido crônico da dermatite atópica induzida por estresse (MOLLANAZAR NK, 2016).

Todos esses mecanismos que ocorrem após uma situação de estresse atuam na liberação de diversos neuropeptídeos e neurotrofinas nas terminações nervosas periféricas, como o $\mathrm{CRH}$ periférico, o fator de crescimento neural (NGF), a substância $P$ e o peptídeo relacionado ao gene da calcitonina (CGRP). Estes atuam em efeitos pró-inflamatórios, principalmente através da ativação de mastócitos, levando a uma inflamação neurogênica (HUNTER HJA, 2015).

Além dos mecanismos imunológicos, o estresse também atua alterando a função de barreira da pele, através da atuação dos glicocorticóides, que impedem a formação de corpos lamelares e diminuem o tamanho e a densidade dos corneodesmossomos. $O$ aumento de glicocorticóides também possui efeito suprimindo a produção de IL-1 $\beta$ e TNF- $\alpha$, citocinas que atuam na cicatrização de feridas (HUNTER HJA, 2015). Algumas doenças que possuem relação com os impactos do estresse sobre a homeostase da pele são a psoríase, a dermatite atópica, o vitiligo, a acne e a alopecia areata (ALEXOPOULOS A, 2016). Existem, ainda, as infecções cutâneas que são reativadas devido ao estresse psicológico, como o herpes (HUNTER HJA, 2015).

A associação de transtornos psiquiátricos com doenças dermatológicas também estão bem documentadas. Alguns exemplos são transtornos depressivos, como depressão grave; transtornos de ansiedade, incluindo fobias; esquizofrenia, abuso de substâncias ilícitas, transtornos bipolares, transtornos de personalidade, transtornos obsessivo-compulsivos, entre outras. Cada uma dessas condições se manifesta na pele de forma diferente, como acne, onicotilomania, prurido generalizado, acrocianose, pêlos semelhantes a lanugens, úlceras, eritemas (TOHID H, et al., 2017).

Além disso, alguns transtornos psiquiátricos podem relacionar com a pele de maneira direta e não apenas por mecanismos neuroquímicos. Isto é, em uma situação de transtorno de ansiedade, por exemplo, o paciente 
pode se arranhar e se ferir manualmente (TOHID H, et al., 2017). Portanto, é essencial que mais estudos devam ser realizados para esclarecimento da relação entre aspectos emocionais e manifestações dermatológicas, uma vez que essa associação é multifatorial.

\section{Impacto psicossocial}

Segundo Orion E e Wolf R (2014) o rosto do ser humano tem grande relevância em praticamente qualquer aspecto da vida: relações interpessoais, apelo sexual, pode afetar o sucesso econômico, entre diversos outros fatores, por isso, sua importância não pode ser subestimada. A aparência física é a primeira impressão no contato humano, é uma importante forma de comunicação, e quando ocorre comprometimento dessas estruturas, principalmente em áreas visíveis, com o surgimento de dermatoses, a autoconfiança fica comprometida, podendo acarretar em algum tipo de sofrimento psíquico (CALVETTI PU, et al., 2017).

As dermatoses geram impacto importante na vida social e emocional, e, de acordo com Calvetti PU, et al. (2017), isso pode ser causado devido aos estigmas gerados pelas lesões dermatológicas no corpo do paciente enfermo.

A maioria das doenças de pele não apresenta um risco direto à vida, entretanto, o comprometimento na aparência física e emocional afeta negativamente a qualidade de vida (CÁRCANO CBM, et al., 2018). Dessa forma, isso pode ser usado como uma justificativa para evitar situações ou relacionamentos levando a diminuição da auto estima e a um isolamento social considerando-se as crenças de que as condições da pele os fazem sentir inferiores (ORION, E E WOLF R, 2014).

De acordo com Taborda MLVV, et al. (2005) o sexo feminino tem maior tendência a apresentar sofrimento psíquico oriundo das dermatoses, o que pode se justificar devido a uma maior preocupação estética por parte das mulheres do que dos homens, e, além disso, ao comparar exclusivamente o impacto negativo do vitiligo e da acne vulgar, a primeira condição se mostrou mais prejudicial psicologicamente, e uma possível justificativa para esse achado seria o fato de que o vitiligo é uma doença que tende a ser mais crônica, além de que o seu tratamento exige muita disciplina e dedicação por parte do paciente, enquanto a acne costuma prevalecer mais na adolescência e tende a regredir naturalmente com o passar dos anos.

Ainda segundo Taborda MLVV, et al. (2005), os primeiros anos de surgimento da doença acarretam em maior prevalência de sofrimento psicológico, provavelmente devido ao impacto inicial gerado pela doença, sendo seguido por uma fase de queda, que pode ser justificado por um período de adaptação, e por fim, depois de anos, há novamente uma elevação na prevalência desse impacto psíquico gerada provavelmente pela frustração com a cronicidade do quadro.

Para mensurar o impacto das lesões de pele na vida do indivíduo, foi desenvolvido o skindex-16, que é um instrumento confiável que avalia esse impacto em três domínios: psicológico/emocional (sentimento do paciente em relação à dermatose), sintomas físicos (como dor ou prurido), e funcionalidade (se o surgimento dessas lesões prejudicou atividades diárias ou relacionamento social) (DIXON LJ, et al., 2018).

Os resultados são obtidos através de um questionário que é aplicado aos indivíduos que possuem essas condições e o observado é que, de fato, há um grande impacto negativo dessas alterações dermatológicas na qualidade de vida dos pacientes. O skindex-16 é uma ferramenta que pode ser utilizada para avaliar pacientes com qualquer condição de pele e auxiliar os médicos em suas práticas clínicas (CÁRCANO CBM, et al., 2018).

Uma grande dificuldade no estudo das psicodermatoses é entender se o surgimento delas é causa ou consequência do estresse psicológico. Já é sabido que algumas condições como albinismo, vitiligo e alopecia areata são condições que levam a um transtorno psíquico secundário, enquanto outras como psoríase, urticária, acne vulgar, etc., são diretamente influenciadas pelo estado emocional, e isso ajuda a direcionar a abordagem clínica mais indicada para aquela situação (TABORDA MLVV, et al., 2005). No entanto, é imprescindível que todos os fatores sejam analisados e abordados na sua integralidade. Muitos distúrbios são provocados ou agravados por estresse, ansiedade, depressão etc., como a acne vulgaris, a rosácea, o herpes labial, a dermatite seborréica, o vitiligo, a dermatite atópica, entre outras condições. Contudo, com uma abordagem ampla e multidisciplinar é possível melhorar a qualidade de vida desses pacientes. 


\section{Abordagem multiprofissional}

A partir da compreensão de que as condições dermatológicas estão diretamente ligadas aos fatores psíquicos e psicológicos do paciente, é de suma importância a abordagem multiprofissional da Psicodermatologia. Essa nova área, consiste em um extenso campo de estudos da dermatologia, psiquiatria e psicologia. Dessa forma, o tratamento dessas condições consiste em tratar o lado emocional do paciente, associado às alterações dermatológicas (FRANÇA K, et al., 2017).

Assim, os dermatologistas desempenham um grande papel no manejo das psicodermatoses uma vez que os pacientes os procuram para tratar somente a pele e frequentemente recusam tratamento psicológico e psiquiátrico (KUHN H, et al., 2017). Por isso, o trato com o paciente e o tratamento a ser empregado exigirá uma atuação mais abrangente do dermatologista, pois ele deverá estar preparado para reconhecer os estados mentais e emocionais que acompanham as dermatoses e dar suporte psicológico ao paciente, a começar por uma relação médico-paciente altamente eficaz, recursos de gerenciamento de estresse e até terapias psicofarmacológicas e psicológicas (AZAMBUJA RD, 2017).

Em um estudo de Markabayeva A, et al. (2019), concluiu-se que os dermatologistas do Cazaquistão, presentes na pesquisa, não obtinham o conhecimento das doenças de pele com componente psicológico, apesar de encontrarem com frequência esse tipo de paciente. É preciso, então, que o dermatologista adquira certas habilidades que vão além do diagnóstico e do manejo das alterações cutâneas. Dessa forma, o especialista que compreende que as queixas emocionais do paciente fazem parte do quadro clínico e tem capacidade para explorá-las e dar-Ihes algum tipo de suporte pode ser mais eficaz em seu tratamento. Mas, as psicodermatoses em muitas vezes apresentam aspectos emocionais que requerem tratamento especializado.

A partir disso o médico dermatologista deve reconhecer e incentivar a necessidade de tratamento psiquiátrico, assim como psicológico. A participação do psiquiatra, quando necessária, é fundamental para o diagnóstico preciso do transtorno de base e o acompanhamento com medicamentos específicos, que exigem conhecimento aprofundado e experiência diária no controle de suas ações e possíveis eventos adversos indesejáveis (AZAMBUJA RD, 2017).

Já as intervenções psicológicas, como treinamento de reversão de hábitos e terapia cognitivocomportamental, resultam em reduções na frequência e severidade da coceira em diferentes dermatoses. Em uma meta-análise recente de Samrao A, et al. (2012), o uso de intervenções psicológicas teve o efeito positivo no tratamento de coceira provocada por dermatoses. O objetivo do treinamento de reversão de hábitos (TRH) é alterar o comportamento disfuncional, ensinando os pacientes a substituir pensamentos e comportamentos negativos, por neutros. No início de sua prática, TRH mostrou ter efeitos positivos no tratamento de alguns transtornos compulsivos relacionados à ansiedade, resultando em uma redução de cerca de $99 \%$ dos hábitos nervosos após 3 semanas. (MOLLANAZAR NK, 2016).

\section{CONSIDERAÇÕES FINAIS}

Considerando a consolidação da medicina psiconeurocutânea a partir do século XX e sua importância nos estudos da conexão mente-pele, torna-se evidente a complexa interação entre o sistema nervoso e a pele. Evidências demonstram que o estresse psicossocial altera a capacidade da pele de responder ao meio externo, através de alterações neuroendócrinas e imunológicas, além disso o estresse crônico atua no desenvolvimento, manutenção e agravamento de doenças tanto físicas quanto mentais. Em decorrência do envolvimento de pele, sistema nervoso e mente, o manejo das doenças psicocutâneas deve integrar profissionais da dermatologia, psiquiatria e psicologia com o intuito de possibilitar o tratamento das psicodermatoses em sua total complexidade.

\section{REFERÊNCIAS}

1. ALEXOPOULOS A, CHROUSOS GP. Stress-related skin disorders. Reviews in Endocrine and Metabolic Disorders, $2016 ; 17(3): 295-304$. 
2. ANTUNES J. Estresse e doença: o que diz a evidência? Psicologia, Saúde \& Doenças, 2019; 20(3): 590-603.

3. AZAMBUJA RD. Dermatologia integrativa: a pele em novo contexto. Anais Brasileiros de Dermatologia, 2000; 75(4): 393-420.

4. AZAMBUJA RD. The need of dermatologists, psychiatrists and psychologists joint care in psychodermatology. Anais Brasileiros de Dermatologia, 2017; 92(1): 63-71.

5. CALVETTI PU, et al. Aspectos biopsicossociais e qualidade de vida de pessoas com dermatoses crónicas. Psicologia, Saúde \& Doenças, 2017; 18(2): 297-307.

6. CÁRCANO CBM, et al. The Brazilian version of Skindex-16 is a valid and reliable instrument to assess the healthrelated quality of life of patients with skin diseases. PloS One, 2018; 13(3): e0194492.

7. DIXON LJ, et al. Stress and skin disease quality of life: the moderating role of anxiety sensitivity social concerns. British Journal of Dermatology, 2018; 178(4): 951-957.

8. FRANÇA K, et al. Pyschodermatology: a trip through history. Anais Brasileiros de Dermatologia, 2013; 88(5): 842843.

9. FRANÇA K, et al. Psychoneurocutaneous medicine: past, present and future. Wiener Medizinische Wochenschrift, 2017; 167: 31-36.

10. GUPTA MA. Psychiatric Dermatology: Management. Clinics in Dermatology, 2018; 36(6): 687-690.

11. HUNTER HJA, et al. The impact of psychosocial stress on healthy skin. Clinical and Experimental Dermatology, 2015; 40(5): 540-546.

12. KUHN H, et al. Psychocutaneous disease: Clinical perspectives. Journal of the American Academy of Dermatology, 2017; 76(5): 779-791.

13. LEE CM, et al. The impact of perceived stress on skin ageing. Journal of the European Academy of Dermatology and Venereology, 2020; 34(1): 54-58.

14. MARKABAYEVA A, et al. Psychodermatology: knowledge, awareness, and attitude of dermatologists in Kazakhstan. International Journal of Dermatology. 2019; 59(4): 105-109.

15. MOLLANAZAR NK, et al. Mediators of Chronic Pruritus in Atopic Dermatitis: Getting the Itch Out?. Clinical Reviews in Allergy \& Immunology, 2016; 51: 263-292.

16. MÜLLER MC, RAMOS DG. Psicodermatologia: uma interface entre psicologia e dermatologia. Psicologia: Ciência e Profissão, 2004; 24(3): 76-81.

17. NOGUEIRA LSC, et al. Vitiligo and emotions. Anais Brasileiros de Dermatologia, 2009; 84(1): 41-45.

18. ORION E, WOLF R. Psychologic factors in the development of facial dermatoses. Clinics in Dermatology, 2014; 32(6): 763-766.

19. PETERS EMJ. Stressed skin? - a molecular psychosomatic update on stress-causes and effects in dermatologic diseases. Journal of the German Society of Dermatology, 2016; 14(3): 233-252.

20. SAMRAO A, et al. A pilot study of an oral phosphodiesterase inhibitor (apremilast) for atopic dermatitis in adults. Archives of Dermatology, 2012; 148:890-897.

21. SHENEFELT PD. Psychodermatological disorders: recognition and treatment. International Journal of Dermatology, 2011; 50(11): 1309-1322.

22. TABORDA MLVV, et al. Avaliação da prevalência de sofrimento psíquico em pacientes com dermatoses do espectro dos transtornos psicocutâneos. Anais Brasileiros de Dermatologia, 2005; 80(4), 351-354.

23. TOHID H, et al. Psychodermatology: An Association of Primary Psychiatric Disorders With Skin. Revista Colombiana de Psiquiatría, 2019; 48(1): 50-57.

24. YANG H, ZHENG J. Influence of stress on the development of psoriasis. Clinical and Experimental Dermatology. 2019; 5(3), 284-288. 